\title{
The effect of wall cooling on a compressible turbulent boundary layer
}

\author{
By R. L. GRAN†, J. E. LEWIS \\ TRW Systems Group, Redondo Beach, California \\ AND T. KUBOTA \\ California Institute of Technology, Pasadena
}

(Received 5 October 1973)

Experimental results are presented for two turbulent boundary-layer experiments conducted at a free-stream Mach number of 4 with wall cooling. The first experiment examines a constant-temperature cold-wall boundary layer subjected to adverse and favourable pressure gradients. It is shown that the boundary-layer data display good agreement with Coles' general composite boundary-layer profile using Van Driest's transformation. Further, the pressuregradient parameter $\beta_{K}$ found in previous studies to correlate adiabatic highspeed data with low-speed data also correlates the present cooled-wall high-speed data. The second experiment treats the response of a constant-pressure highspeed boundary layer to a near step change in wall temperature. It is found that the growth rate of the thermal boundary layer within the existing turbulent boundary layer varies considerably depending upon the direction of the wall temperature change. For the case of an initially cooled boundary layer flowing onto a wall near the recovery temperature, it is found that $\delta_{T} \sim x$ whereas the case of an adiabatic boundary layer flowing onto a cooled wall gives $\delta_{T} \sim x^{\frac{1}{2}}$. The apparent origin of the thermal boundary layer also changes considerably, which is accounted for by the variation in sublayer thicknesses and growth rates within the sublayer.

\section{Introduction}

In recent years there has been considerable progress towards delineating the effects of both adverse and favourable pressure gradients on the development of a turbulent boundary layer. The effect on low-speed flows, for example, was treated at the AFOSR-Stanford Conference as reported by Coles \& Hirst (1969). One very important conclusion of this conference was that the majority of experimental data were adequately described by a universal composite boundarylayer profile first suggested by Coles (1956):

$$
\frac{U}{U_{\tau}}=f\left(\frac{y U_{\tau}}{\nu}\right)+\frac{\tilde{\pi}}{\kappa} W\left(\frac{y}{\delta}\right)
$$

$\dagger$ Present address: Flow Research, Inc., Los Angeles, California.

$\ddagger$ Present address: Techmate, Inc., Torrance, California. 
where $U$ is the velocity at the location $y, U_{\tau}=\left(\tau_{w} / \rho\right)^{\frac{1}{2}}$ is the friction velocity, $\tau_{w}$ is the wall shear stress, $\rho$ is the fluid density, $\nu$ is the kinematic viscosity, $\tilde{\pi}$ is the wake strength parameter, $\delta$ is the boundary-layer thickness, $\kappa$ is Kármán's constant, taken as 0.41 , and finally $W$ is the wake function. Further, it is well known that the function $f$ appearing in (1) has the form

$$
f \sim\left\{\begin{array}{l}
y U_{\tau} / \nu \text { as } y U_{\tau} / \nu \rightarrow 0 \\
\kappa^{-1} \ln \left(y U_{\tau} / \nu\right)+C \text { for } y U_{\tau} / \nu \geqslant 50,
\end{array}\right\}
$$

with the constant $C$ taken as $5 \cdot 0$, while the wake function $W$ is closely represented by

$$
W \sim 2 \sin ^{2}(\pi y / 2 \delta) .
$$

Both of these functions are apparently universal in that they are unaffected by pressure gradients. The effect of the pressure gradient appears only in the two parameters $U_{\tau} / U_{e}$ (where $U_{e}$ is the free-stream velocity) and $\tilde{\pi}$, which are found to be reasonably well correlated using the Clauser (1956) parameter

$$
\beta \equiv\left(\delta^{*} / \tau_{w}\right) d p / d x
$$

where $\delta^{*}$ is the boundary-layer displacement thickness,

$$
\delta^{*} \equiv \int_{0}^{\infty}\left(1-U / U_{e}\right) d y
$$

It is quite natural that the success in describing low-speed turbulent boundary layers has provided the impetus for similarly describing high-speed or compressible turbulent boundary layers. The most obvious change brought on by highspeed flow is the fact that the density is no longer uniform across the boundary layer. To account for this, Van Driest (1951) suggested a velocity transformation $\left(U \rightarrow U^{*}\right.$ ) which employs the Crocco relation (i.e., $T_{T} \propto U$, where $T_{T}$ is the total temperature $) \dagger$ to account for the density variation. Maise \& McDonald (1967) were further able to show that for the adiabatic flat plate $(d p / d x=0$ and $T_{w}=T_{R}$, where $T_{w}$ is the wall temperature and $T_{R}$ is the recovery temperature) this transformation did indeed reduce compressible data to the form given in (1). Matthews, Childs \& Paynter (1970) successfully applied this to adiabatic boundary layers recovering from a strong perturbation (such as passing through a shock wave) and Chen (1972) extended Maise \& McDonald's correlation to nonadiabatic rough walls. Furthermore, Lewis, Gran \& Kubota (1972) reported experimental adiabatic-wall data that not only supported Van Driest's transformation for adverse and favourable pressure gradients but also showed that

† The derivation of the Crocco relation $\left(T_{T}-T_{T_{e}}\right) /\left(T_{w}-T_{T_{e}}\right)=U / U_{e}$ assumes that the laminar Prandtl number is unity and that there is no pressure gradient. The effect of a pressure gradient on the total temperature distribution has been treated theoretically by Alber \& Coats (1969) for incompressible flows using an approach similar to Mellor \& Gibson's (1966) solution for the velocity profile. Alber \& Coats show a noticeable change in the Crocco relation for both adverse and favourable pressure gradients. This change was not observed in the present experiments and thus the Crocco relation given above was assumed valid. 
the dependence of $U_{\tau}$ and $\tilde{\pi}$ on the local pressure gradient was the same as for low-speed flows if the parameter $\beta_{K}=\left(\delta_{K}^{*} / \tau_{w}\right)(d p / d x)$, where

was used.

$$
\delta_{K}^{*} \equiv \int_{0}^{\infty}\left(1-U / U_{\infty}\right) d y
$$

In order to extend the applicability of Van Driest's transformation and the correlations of $U_{\tau}$ and $\tilde{\pi}$ with $\beta_{K}$ an experiment was performed where the model walls were highly cooled. One purpose of this paper is to describe the results of this experiment and compare these with the existing correlations.

The second purpose of this paper is to describe another experiment which treats the relaxation of a compressible flat-plate boundary layer after a sudden change in boundary conditions. Physically, this change is manifested by a step change in the wall temperature. Thus, a thermal boundary layer develops within the existing turbulent boundary layer. In this experiment the external flow is constant at $M_{\infty}=4$ and the change in wall temperature occurs over a distance of 3-4 boundary-layer thicknesses.

Previous investigations dealing with the response of a low-speed turbulent boundary layer to a sudden change in boundary conditions have been performed by changing the surface roughness (e.g. Antonia \& Luxton 1971, 1972). Townsend (1965) has considered this problem theoretically and has shown that the new momentum boundary layer developing within the existing boundary layer grows like $x$ far downstream of the discontinuity. This result was not verified by Antonia \& Luxton, who reported smaller growth rates, especially for the case where the flow proceeds from a rough wall onto a smooth wall. Because of this discrepancy between experiment and theory at low speeds coupled with the possible effects brought on by compressibility, the present data are considered important.

In the following sections, descriptions of the experimental facility and windtunnel model are first given followed by the data reduction procedure. The results for the experiment with constant wall temperature and a pressure gradient are then presented and compared with adiabatic-wall data and lowspeed data. Finally, the results for the response of a constant-pressure boundary layer to a step change in wall temperature are given.

\section{Experimental procedure}

The experiments described herein were conducted in the $40 \times 40$ in. supersonic wind tunnel $A$ of the von Kármán Gas Dynamics Facility at the Arnold Engineering Development Center, Arnold Air Force Station, Tennessee. The free-stream Mach number was 4.0 and the free-stream unit Reynolds number was $0.5 \times 10^{6} \mathrm{in} .^{-1}$, corresponding to a total pressure of $4.9 \mathrm{~atm}$ and total temperature of $45^{\circ} \mathrm{C}$.

The wind-tunnel model is the same as that described in Lewis et al. (1972) except that copper cooling coils were attached to the exterior surface in order to cool the model wall. The model, shown in figure 1, consists of basically two parts: 


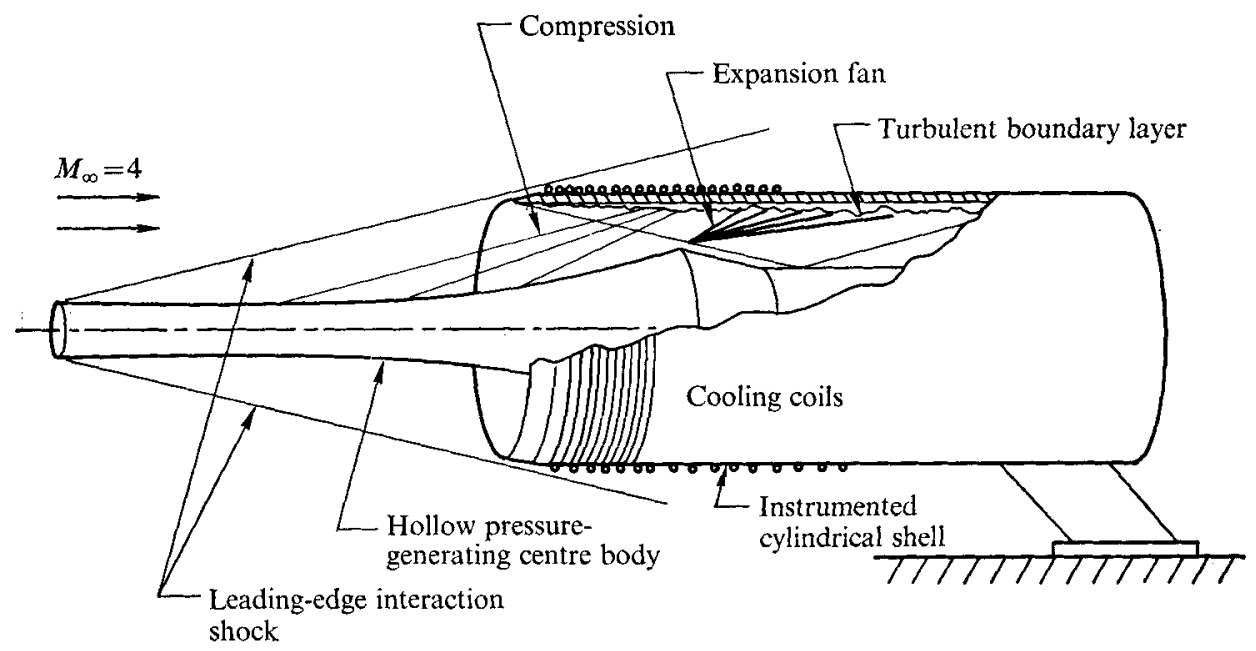

Figure 1. Wind-tunnel model.

an outer shell and an inner pressure-generating body. The outer shell is a hollow cylinder with a diameter of $20 \mathrm{in}$. and a length of $50 \mathrm{in}$.; the boundary layer formed on the inner surface of this shell was studied. The inner body is shaped to give a prescribed pressure distribution along the wall of the outer shell. This geometry is particularly advantageous for several reasons: first, the problem of end effects associated with planar models is eliminated and second, the large constant radius $\left(\delta / r_{w} \lesssim 0 \cdot 07\right)$ minimizes the effects of transverse curvature and eliminates effects associated with streamwise surface curvature.

The temperature of the outer shell was depressed by pumping liquid nitrogen through the cooling coils, which were segmented and interlaced to prevent boiling and produce a nearly uniform wall temperature. This coil segmenting also permitted water to be pumped through either the fore or aft set of coils while liquid nitrogen was pumped through the remaining set. This feature allowed a near step change in the model wall temperature.

The measurements obtained during the course of the experiment were distributions of wall temperature, wall pressure and heat flux, $\dagger$ along with boundarylayer surveys at selected stations. These surveys consisted of Pitot-pressure and total-temperature measurements. Additional details regarding the wind tunnel, the model or the instrumentation can be found in Hahn \& Lutz (1971).

\section{Data reduction}

\subsection{General}

The Pitot pressure and the measured wall pressure at a given survey location were used to calculate the Mach number profile $M(y)$ across the boundary layer. The static pressure at the boundary-layer edge computed from the edge Pitot

$\dagger$ The wall heat flux was obtained using an asymptotic calorimeter. The heat-transfer data presented here are uncorrected for any sensor/boundary-layer interaction because of a frost layer which formed on the model wall (discussed in $\$ 3.1$ ). For a clean surface this interaction could lead to an actual wall heat flux some 10-20\% above the measured heat flux. 


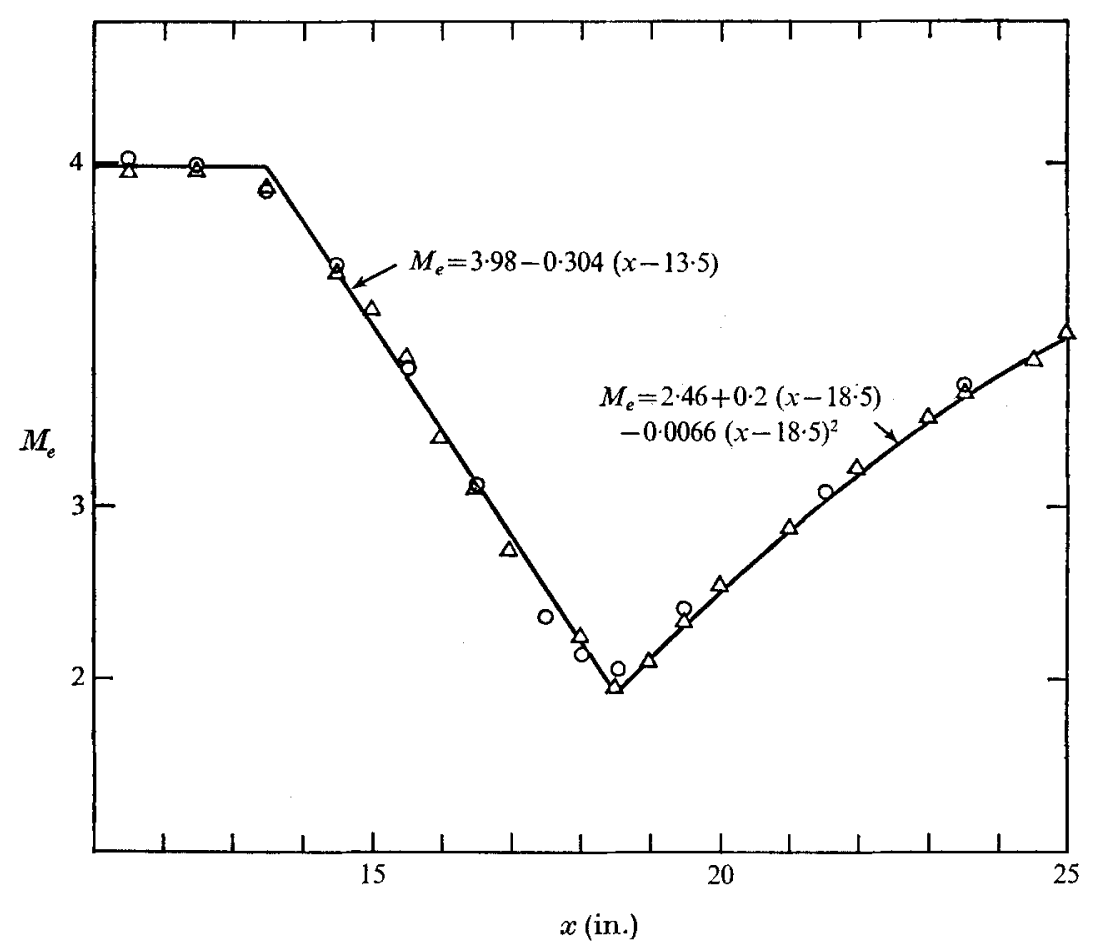

Figure 2. Boundary-layer-edge Mach number distribution. $\longrightarrow$, analytical fit to data; $\bigcirc$, Pitot-isentropic; $\triangle$, wall pressure.

pressure agreed quite well (randomly within $\pm 10 \%$ as inferred from the edge Mach number distribution of figure 2) with the measured wall pressure, indicating that the static pressure across the boundary layer was nearly constant. The velocity profile was then calculated from $M(y)$ and the total-temperature measurements $T_{T}(y)$.

The cooling of the model with liquid nitrogen did, in fact, cause a layer of frost to form on the model wall. This frost layer grew in thickness at an estimated rate of about $0.001 \mathrm{in} . / \mathrm{min}$ and, because of this, data were only taken over $20 \mathrm{~min}$ periods (thus, $y_{f} / \delta \lesssim 0 \cdot 1$ ), after which the cooling was turned off and the model 'defrosted'. Over a 20 min period the measured wall heat flux was observed to decrease by $10-15 \%$. Even though precautions were taken to keep the frost thickness small, a correction to the probe position with respect to the 'wall' was necessary using the chronological test record and the assumed growth rate.

The frost layer had the additional effect of causing the effective wall temperature 'seen' by the boundary layer to be greater than the measured wall temperature $T_{w M}$ of $0 \cdot 30( \pm 0 \cdot 02) T_{T e}$. To illustrate this, a plot of the measured total temperature $v s$. the measured velocity ratio obtained for a number of flat-plate survey locations is shown in figure 3 . Also shown is the Crocco relation

$$
\left(T_{T}-T_{w}\right) /\left(T_{T e}-T_{w}\right)=U / U_{e}
$$




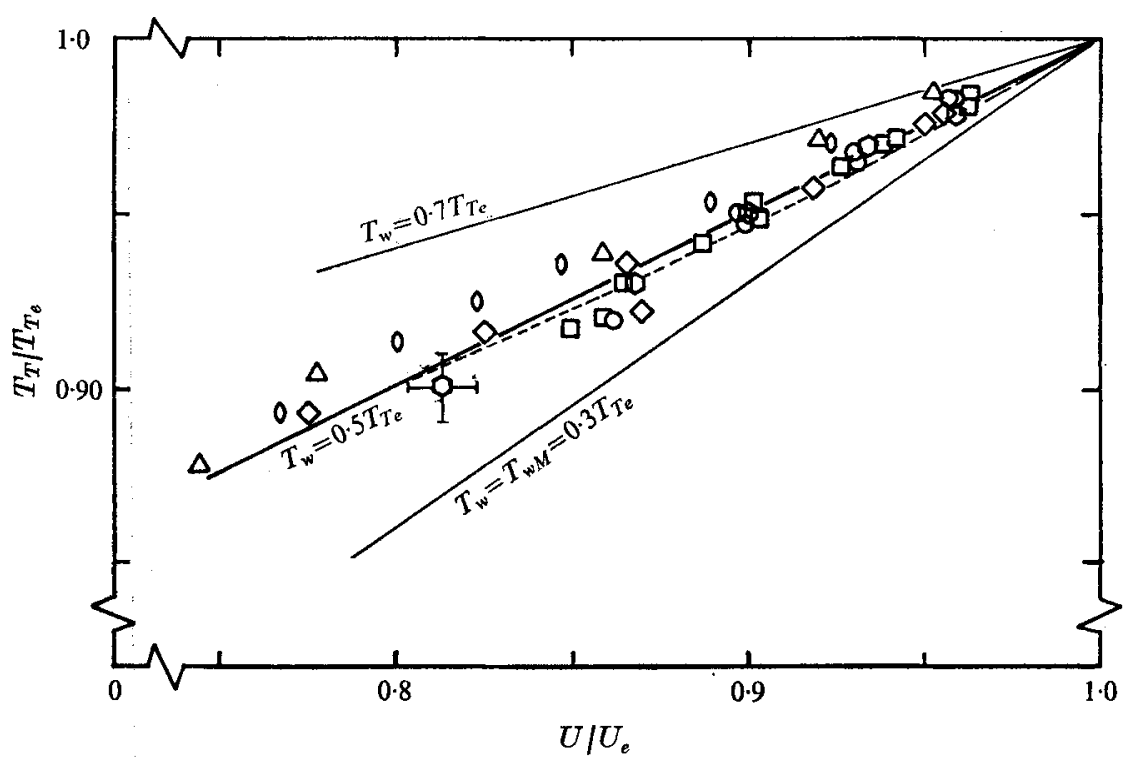

Figure 3. Measured total temperature vs. measured velocity ratio for a flat plate. $\bigcirc, x=12.5 ; \square, x=13 ; \diamond, x=19.5 ; \triangle, x=21.5 ; 0, x=24.5 ; 0, x=25.5 ;$ Crocco law for $T_{w} / T_{T_{e}}=0.3,0.5,0.7 ; \ldots$, quadratic law for $T_{w} / T_{T_{e}}=0.72 ;|-|$, error bounds of measured data.

for several values of the effective wall temperature, represented by the solid lines, and a quadratic total-temperature/velocity relation (which approximates data. for wind-tunnel wall boundary layers)

$$
\left(T_{T}-T_{w}\right) /\left(T_{T e}-T_{w}\right)=\left(U / U_{e}\right)^{2},
$$

represented by the dashed line. A comparison between the data and these approximations yields two tentative conclusions: (i) if the Crocco relation is assumed valid over the entire boundary-layer thickness, the effective wall temperature $T_{w}$ is nearer to $0 \cdot 5 T_{T e}$ than to $T_{w M}$ and (ii) if the quadratic totaltemperature/velocity relation is used, an even higher effective wall temperature of $0.72 T_{T e}$ is required to match the data.

In order to make an independent estimate of the effective wall temperature it is noted that the measured model wall temperature $T_{w M}=0 \cdot 3 T_{T e}$ is less than the sublimination temperature for both carbon dioxide $\left(0 \cdot 35 T_{T e}\right.$ at a molar concentration of $\left.3 \times 10^{-4}\right)$ and water vapour $\left(0 \cdot 67-0 \cdot 69 T_{T e}\right.$, which corresponds to a tunnel air dew-point temperature range between $-27^{\circ} \mathrm{F}$ and $-10^{\circ} \mathrm{F}$ or a molar concentration between $3 \times 10^{-4}$ and $8 \times 10^{-4}$ ). Thus, the frost layer could contain both solid $\mathrm{CO}_{2}$ and water. However, some of the water vapour probably condenses in the free stream prior to encountering the cold model wall (the condensation Mach number for water vapour is estimated to be about 2 according to Wegner \& Mack (1958) and the peak temperature in the boundary layer is insufficient to cause evaporation of the condensed water particles). Thus, the frost is believed to be composed principally of $\mathrm{CO}_{2}$ with a small amount of water. The surface temperature of the frost mixture is raised appreciably above the $\mathrm{CO}_{2}$ 

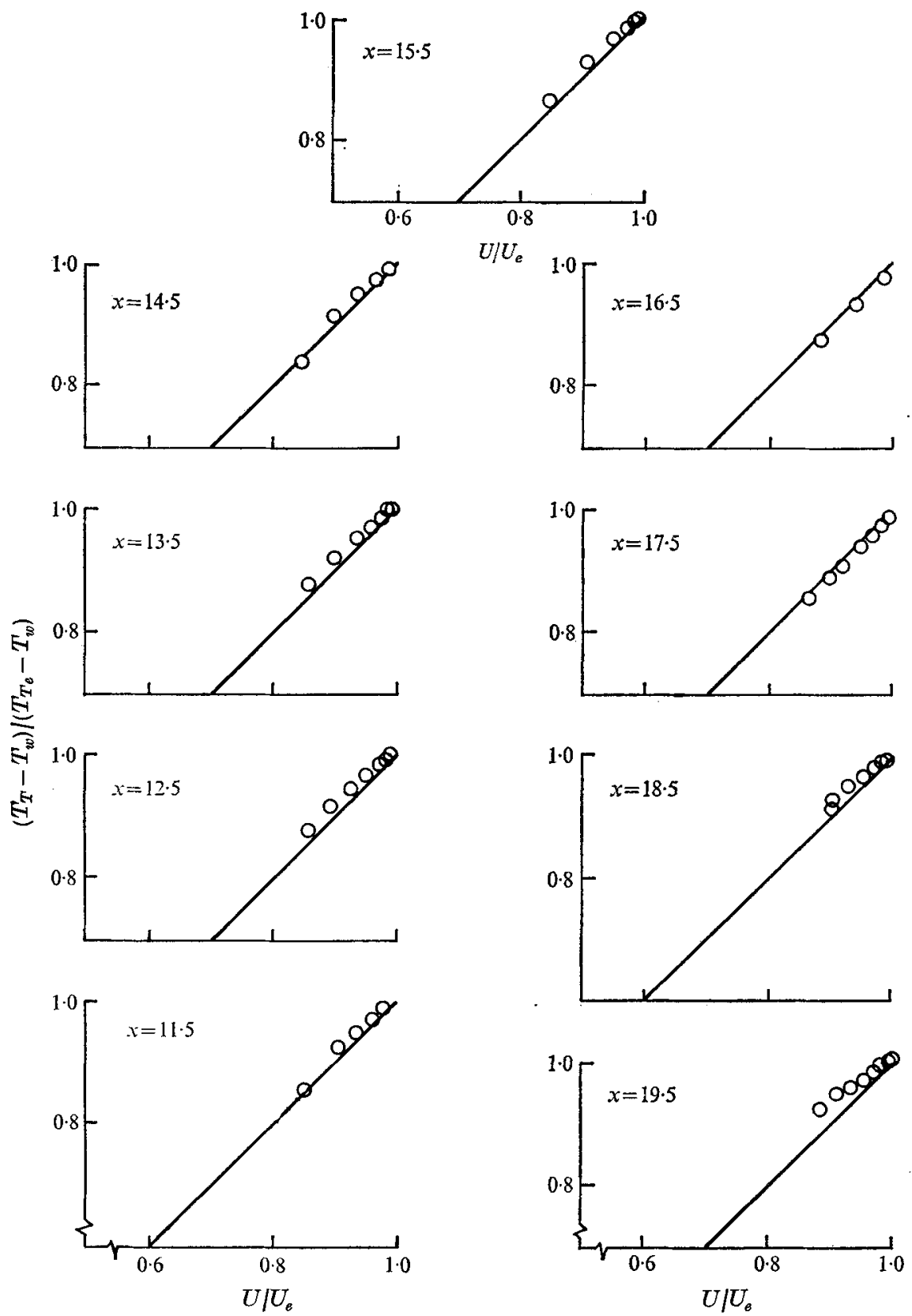

FraURe 4. Effect of pressure gradient on profile of total temperature vs. velocity ratio. - Crocco relation; $\mathrm{O}$, measured point.

sublimation temperature by the simultaneous condensation of water despite the difference in condensation rates as shown in a related example by Bird, Stewart \& Lightfoot (1963, p. 586). Even a trace amount can cause the surface temperature to be the mean of the two sublimation temperatures, which for the present experiment is very near to $0 \cdot 5 T_{T e}$. Because of this estimate and the concurrence 
with the wall temperature inferred from the Crocco relation, it has been assumed that the Crocco relation holds throughout the boundary layer at least for zeropressure-gradient regions and that the effective wall temperature is $0.5 T_{T \ell}$.

To check the Crocco relation in regions of non-constant pressure totaltemperature data are plotted $v s$. velocity data in figure 4 for both adverse $(11 \cdot 5 \leqslant x \leqslant 17 \cdot 5)$ and favourable $(18 \cdot 5 \leqslant x)$ pressure gradients. No significant deviation from the Crocco relation is observed. This fact is important in that the boundary-layer velocity-profile transformation described below employs the Crocco relation.

Having computed the velocity and total-temperature distributions, the evaluation of the local boundary-layer integral properties was effected by an integration of the measured data excluding the sublayer. This sublayer contribution was evaluated using Coles' (1953) tabulated sublayer function for the region $0<y U_{\tau} / \nu_{w} \lesssim 50$. This is expressed as

$$
U^{*} / U_{\tau}=f\left(y U_{\tau} / \nu_{w}\right)
$$

where $U^{*}\left(U_{e}, M_{e}, T_{w}, T_{T e}\right)$ is the Van Driest (1951) transformed velocity, which employs the Crocco relation discussed above, and $U_{\tau}$ is the friction velocity $\left[=\left(\tau_{w} / \rho_{w}\right)^{\frac{1}{2}}, \tau_{w}\right.$ being the wall stress and $\rho_{w}$ the density at the wall $]$.

The local pressure gradient $d p / d x$ used in the calculation of $\beta_{K}\left[\equiv\left(\delta_{K}^{*} / \tau_{w}\right) d p / d x\right.$, where $\delta_{K}^{*}$ is defined in $\left.\S 1\right]$ was evaluated using an analytic expression for the edge Mach number distribution $M_{e}(x)$. The experimental values of $M_{e}(x)$ computed from the boundary-layer-edge Pitot pressure and from the measured wall pressure are shown in figure 2 . In addition, the selected analytic expression for $M_{\varepsilon}(x)$ is also shown. $\dagger$

\subsection{Profile interpretation}

The velocity-profile data at each station were handled in the manner described in Lewis et al. (1972) after allowance had been made for the inferred wall temperature and frost thickness as discussed above. Basically, this procedure fits the transformed data to the two-parameter composite boundary-layer profile

$$
\frac{U^{*}}{U_{\tau}}=\frac{1}{\kappa} \ln \frac{y U_{\tau}}{\nu_{w}}+C+\frac{\tilde{n}}{\kappa} W\left(\frac{y}{\delta}\right)
$$

The two parameters obtained from this procedure are the skin-friction coefficient and the local boundary-layer thickness. The wake strength parameter $\tilde{\pi}$ is obtained in terms of $U_{\tau} / U_{e}\left[=\left(\frac{1}{2} C_{f} T_{w} / T_{e}\right)^{\frac{1}{2}}\right]$ and $\delta$ by evaluating (7) at the boundary-layer edge. $\$$

† The expression for $M_{e}(x)$ is identical to that used in Lewis et al. (1972) for the adiabaticwall case since the same internal model configuration was used here. The footnote in this reference regarding the analytic fit in the region around $x=13.5$ and $x=15.8$ is also applicable here in that some rounding of the sharp corners is evident in the data.

\#t the suggestion of one of the referees a similar fit was made using a velocity transformation based upon the quadratic total-temperature/velocity relation (5) for the flatplate measurements (and with $T_{w}=0.72 T_{T e}$ ). This procedure gave results for $C_{f}, \delta$ and $\#$ very close to those computed above. Note, however, that the heat-transfer coefficient defined in (8) would be almost twice as large as the values shown in figure 4, which, in turn, would cause the Reynolds analogy factor $2 C_{H} / C_{f}$ to be appreciably larger than the accepted value of 1.0. This has been interpreted as an additional justification for the use of the Croceo relation. 


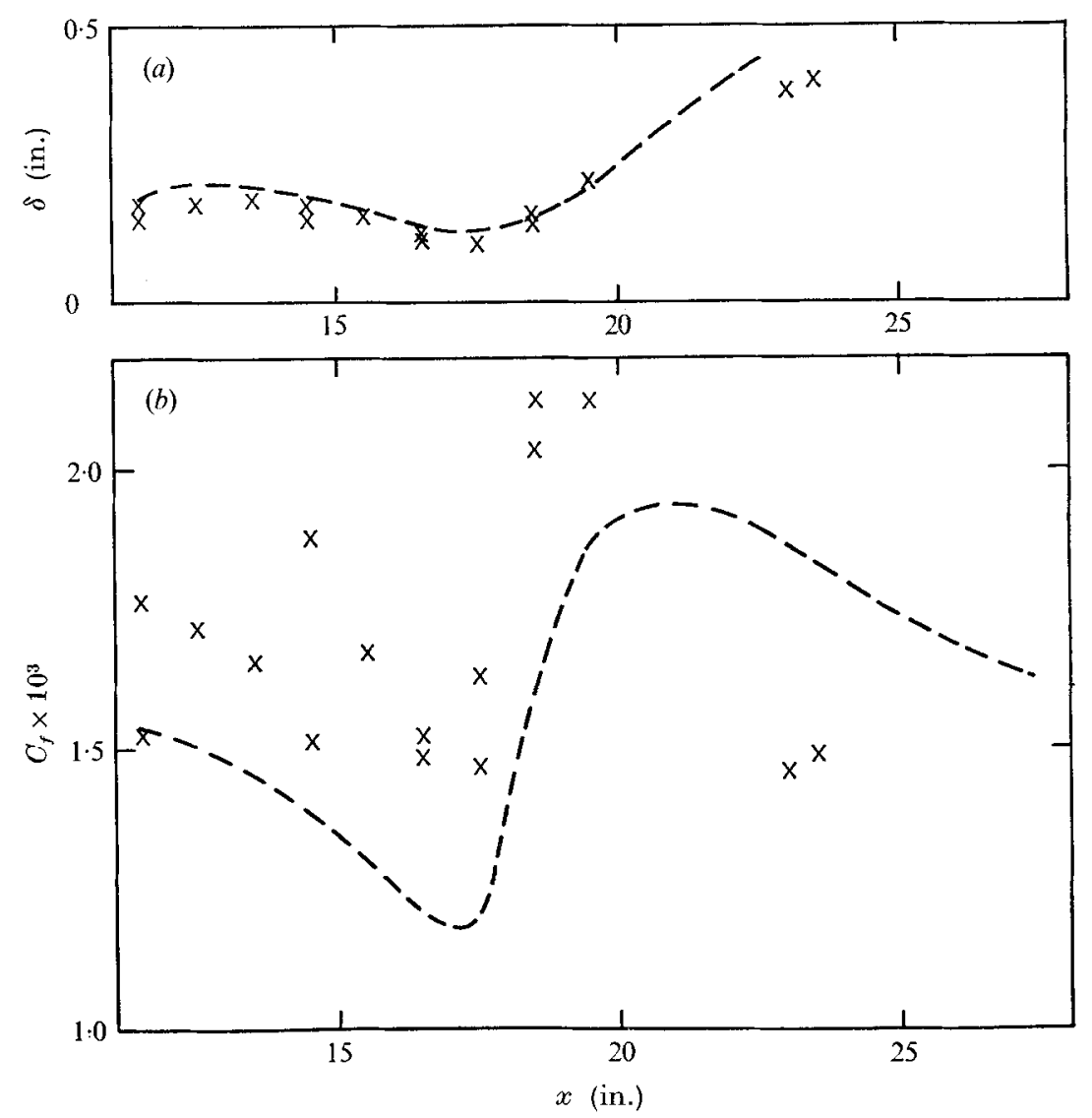

Figures $5(a, b)$. For legend see next page.

\section{Experimental pressure-gradient results}

The development of the boundary layer along the model is shown in figure 5 . Shown are the boundary-layer thickness, the Reynolds number based on the momentum thickness, the calculated wall friction coefficient $C_{f}\left(\equiv \tau_{w} / \frac{1}{2} \rho_{e} U_{e}^{2}\right)$ and the measured heat-transfer coefficient

$$
C_{h} \equiv \dot{q}_{\text {wall }} / \rho_{e} U_{e} C_{p}\left(T_{r}-T_{w}\right),
$$

where $C_{p}$ is the specific heat at constant pressure and $T_{r}$ is the recovery temperature $\approx 0.91 T_{T e}$. For comparison, the adiabatic-wall results are also shown for the first three graphs of figure 5. Shown with the heat-transfer measurements is a line representing a mean $\frac{1}{2} C_{f}$ in order to check the Reynolds analogy factor $R \equiv 2 C_{h} / C_{f}$. Although there is a paucity of data in the region of adverse pressure gradient $(13 \cdot 5 \leqslant x \leqslant 18 \cdot 5)$, it is clear that $R$ is close to unity throughout this region. In the region of favourable pressure gradient and within the accuracy of the data the heat-transfer coefficient $C_{h}$ exceeds $\frac{1}{2} C_{f}$, in contrast to theoretical solutions (e.g. Alber \& Coats), which show a decrease in $R$ for equilibrium 

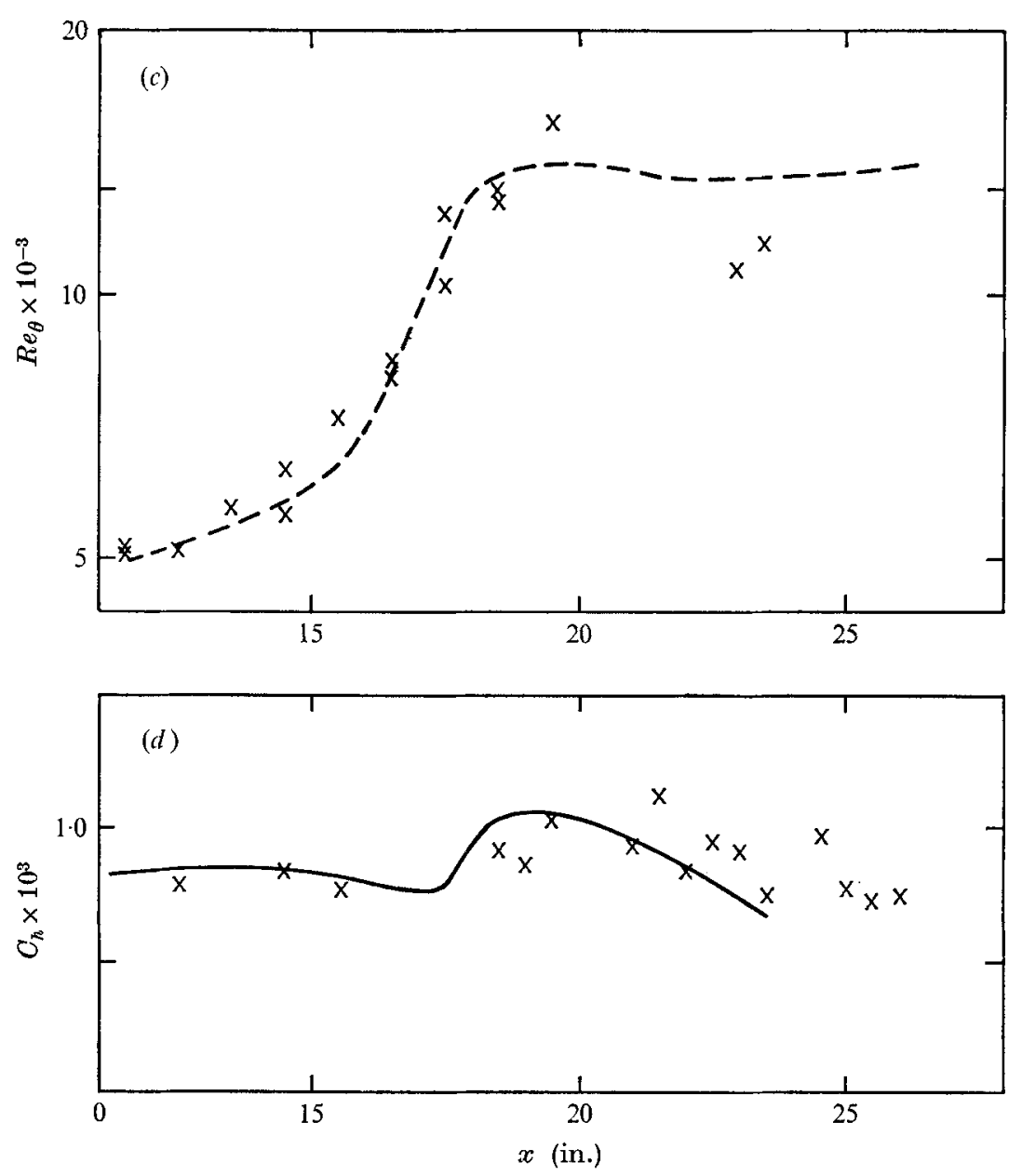

FIqURE 5. Boundary-layer characteristics. (a) Boundary-layer thickness. (b) Skin-friction coefficient. (c) Reynolds number based upon momentum thickness. (d) Heat-transfer coefficient. ......, results for adiabatic wall; - - $\frac{1}{2} C_{f}$.

boundary layers in favourable pressure gradients. The local pressure-gradient parameter $\beta_{K}$ and the 'wake strength' parameter $\tilde{\pi}$ are shown in figure 6 along with a line representing the corresponding adiabatic-wall data taken from Lewis et al. Note that the cold wall results in a weaker pressure gradient because $\delta_{K}^{*}$ is smaller and the wall shear stress is somewhat higher.

The integrated streamwise momentum equation given by

$$
\left(\frac{q_{e} \theta}{q_{e_{0}} \theta_{0}}-1\right)-\frac{1}{2} \int_{p_{0}}^{p} \frac{\delta^{*}}{\theta_{0}} \frac{d p}{q_{e_{0}}}=\frac{1}{2} \int_{x_{0}}^{x} \frac{\tau_{w}}{q_{e_{0}}} \frac{d x}{\theta_{0}}
$$

is shown in figure 7 , in which the left-hand side of $(9)$ is plotted against the righthand side. The $30 \%$ discrepancy in the experimental momentum balance is greater than that found for the corresponding adiabatic case. This discrepancy could be the result of the wall stress calculated using the profile fitting procedure 


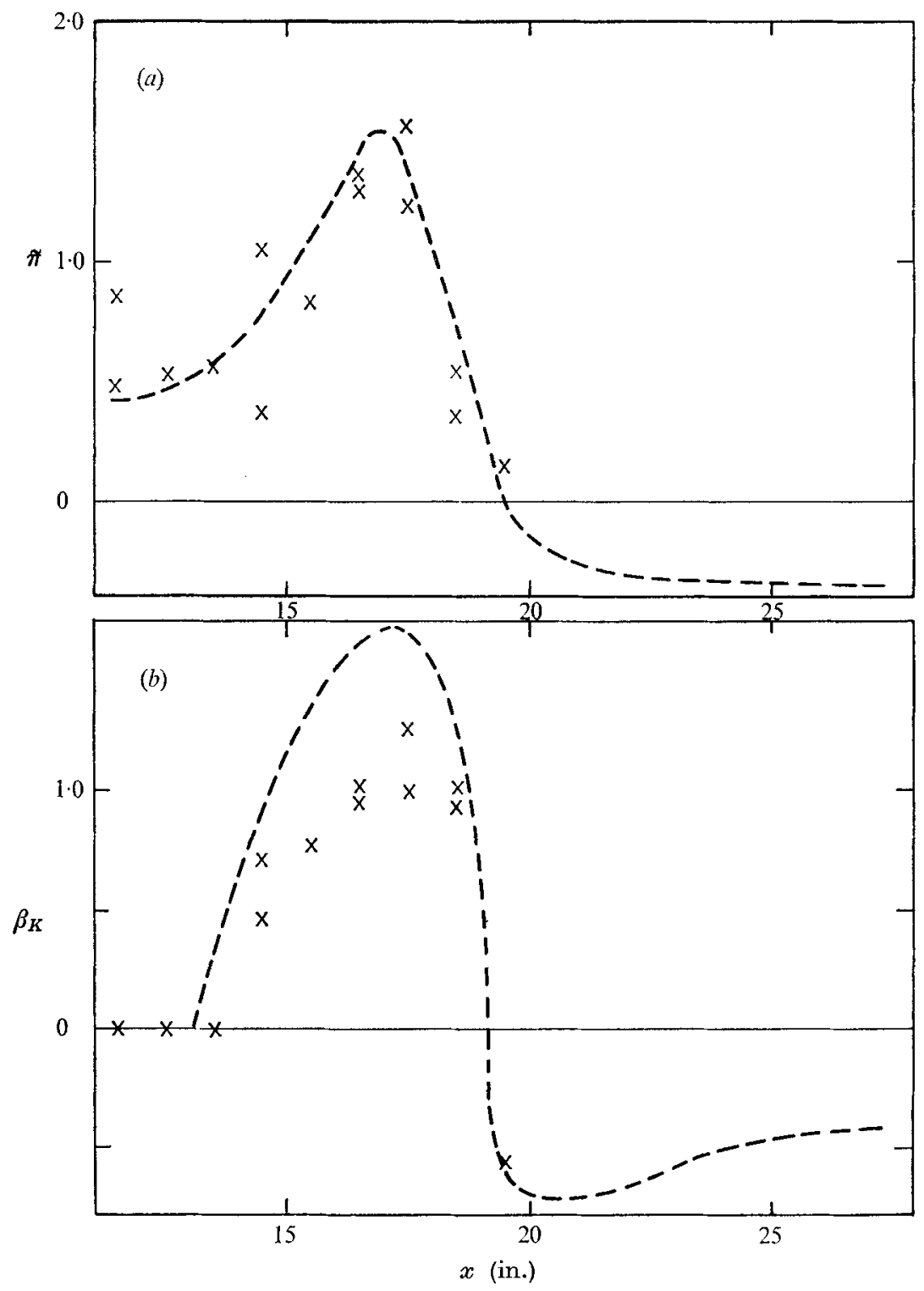

Figure 6. Boundary-layer characteristics. $(a)$ Wake strength parameter $\#$. (b) Pressure-gradient parameter $\beta_{K}$. ......, results for adiabatic wall.

described in $\$ 3.2$ being too low. Stanton-tube measurements of wall stress could not be used to check this because of the frost formation.

The transformed velocity profiles are shown in law-of-the-wall form in figure 8 for the region of zero and adverse pressure gradient and for the region of favourable pressure gradient. The wake function defined as

$$
W\left(\frac{y}{\delta}\right)=\frac{\kappa}{\check{\pi}}\left\{\frac{U_{e}^{*}}{U_{\tau}}-\frac{1}{\kappa} \ln \frac{y U_{\tau}}{v_{w}}-C\right\}
$$




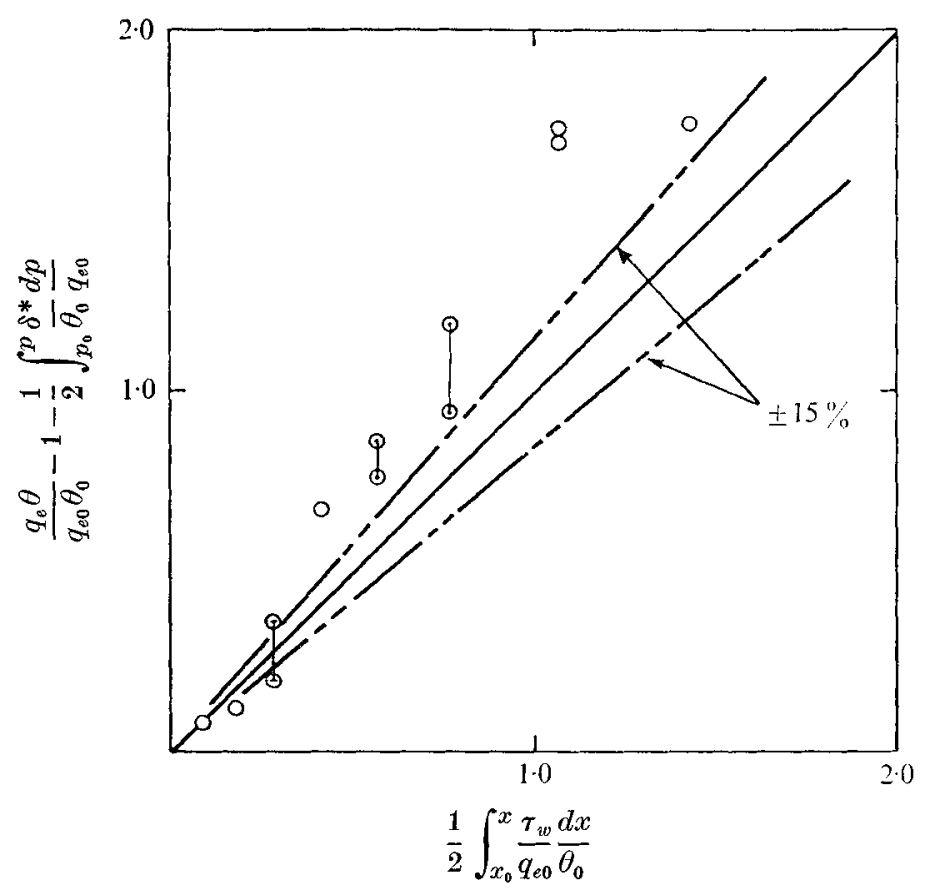

Figure 7. Streamwise momentum balance._-_, line of perfect agreement; $-\cdots-, \pm 15 \%$ error limits.

is shown in figure 9 for the regions of adverse and zero pressure gradient along with the analytical approximation (3). In the region of favourable pressure gradient the significant difference between the experimental values of $W$ (not shown) and the assumed functional form (3) is much like that shown by the previous adiabatic-wall data in Lewis et al. (1972). Undoubtedly, the conjectured non-equilibrium effects discussed in Lewis et al. are present here also. Insufficient data in the region of favourable pressure gradient in the present experiment precludes further elaboration on this phenomenon.

The use of Coles' composite boundary-layer profile (7) for the calculation of boundary-layer development requires that the influence of the pressure gradient on the two parameters $C_{f}$ (or $U_{\tau}$ ) and $\tilde{\pi}$ be known. The extension of this to compressible flows (using Van Driest's transformation) does not alter this requirement. It was previously found that the pressure-gradient parameter $\beta_{K}$ correlates the influence of the pressure gradient in the low-speed and the adiabatic-wall high-speed cases [i.e. no Mach number dependence exists for $\left.\tilde{\pi}\left(\beta_{K}\right)\right]$. One purpose of the present investigation is to test this comparison for the cold-wall high-speed case.

The influence of the pressure gradient on the local friction coefficient $C_{f}$ is shown in figure $10(a)$. An attempt to remove the effects of Mach number and Reynolds number has been made by normalizing the measured

$$
C_{f}\left(M_{e}, R e_{\theta}, T_{w} / T_{r}, \beta_{K}\right)
$$




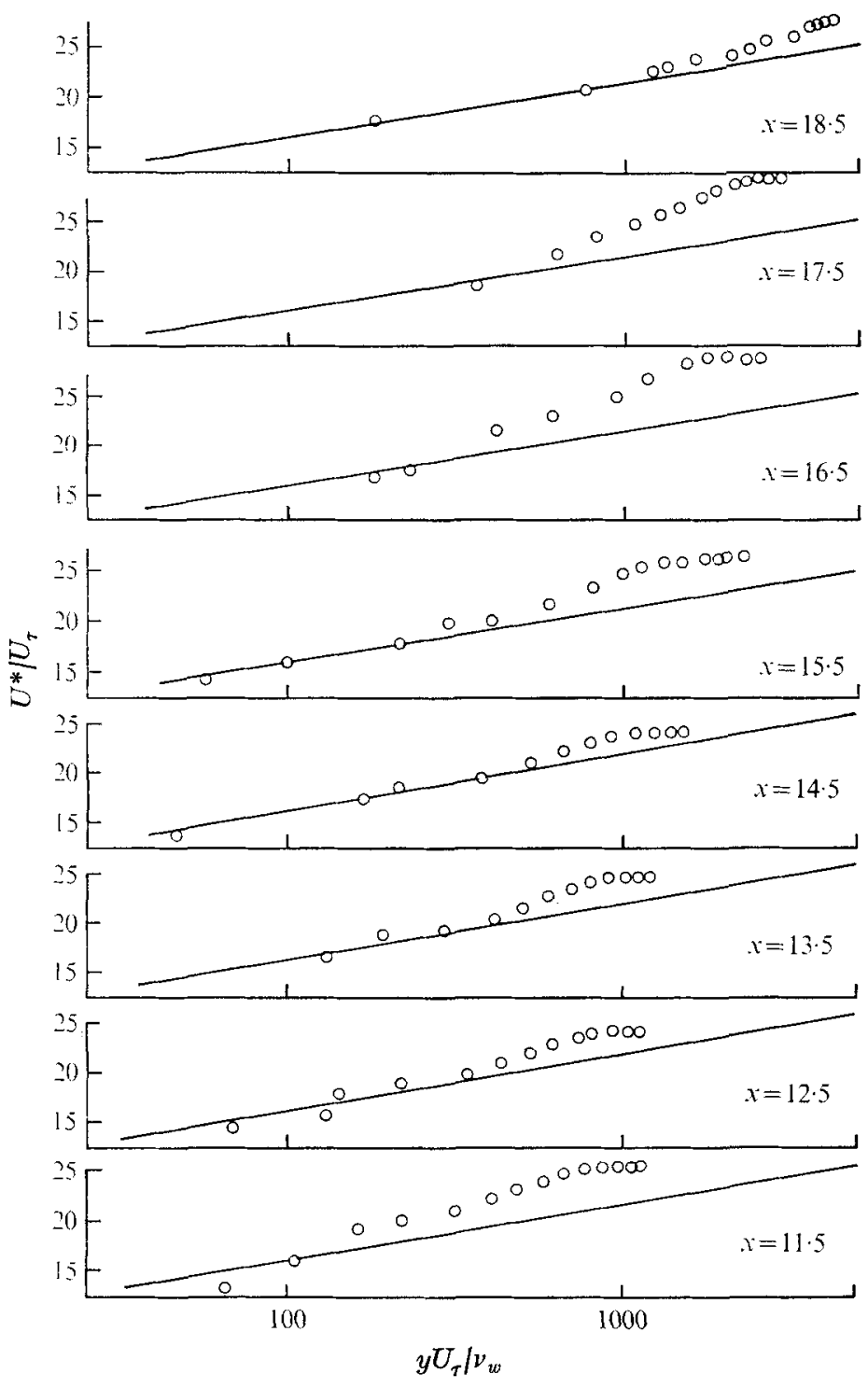

Figure 8. Transformed law-of-the-wall velocity profiles. , law of the wall (typical).

with the calculated flat-plate value at the same $M_{e}, R e_{\theta}$, and $T_{w} / T_{r} . \dagger$ Also shown for comparison in figure $10(a)$ is the correlation between the low-speed and adiabatic-wall cases from Lewis et al. The cold-wall data, though somewhat scattered, display the same trend as the comparison cases.

The effect of the pressure gradient on the so-called wake strength parameter $\tilde{\pi}$ is shown in figure $10(b)$ along with the correlation between the low-speed and

$\dagger$ The calculated flat-plate friction coefficient was computed using the Van Driest II formulation of Hopkins \& Inouye (1971). 


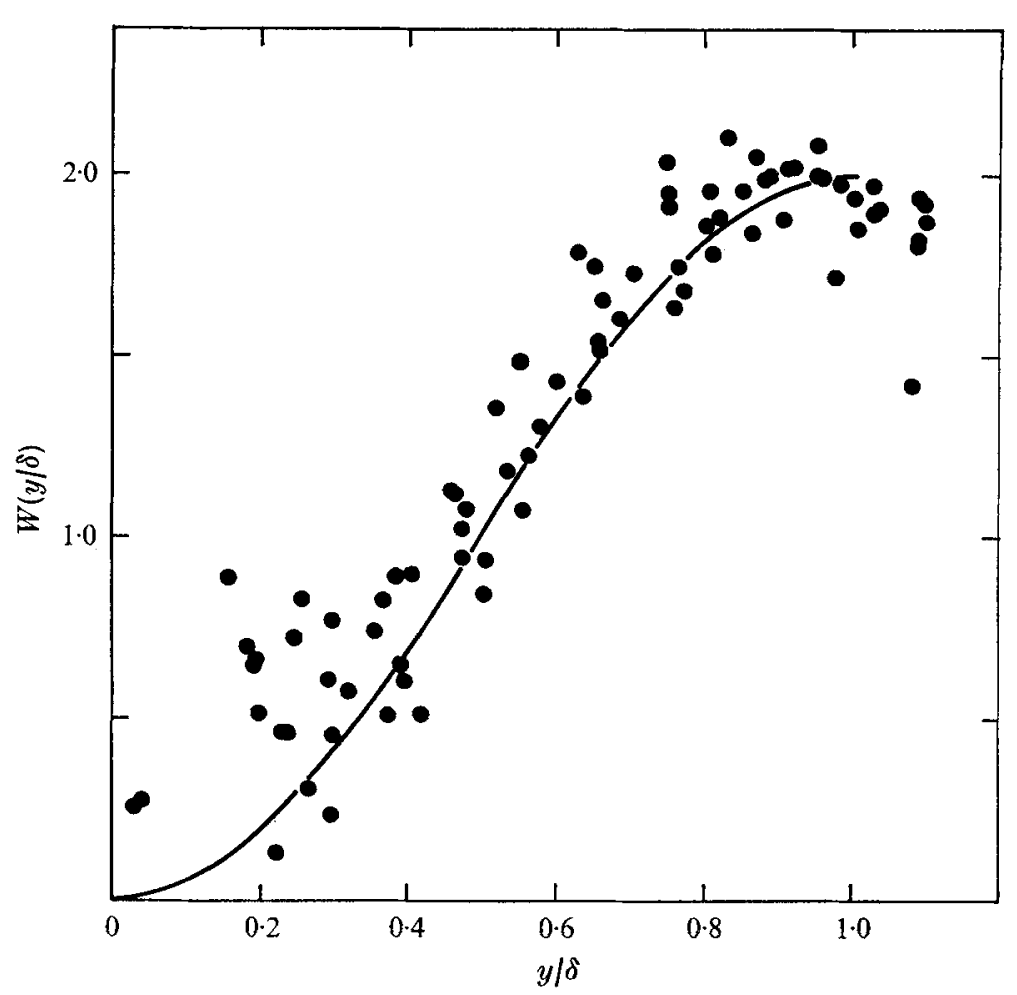

Figure 9. Coles' wake function. - $-\sin ^{2}(\pi y / 2 \delta)$.

adiabatic high-speed data. Again, the cold-wall high-speed $\tilde{\pi}\left(\beta_{K}\right)$ relation is as good as either of the comparison cases. Further, the value of $\beta \equiv\left(\delta^{*} / \tau_{w}\right) d p / d x$ is roughly twice the value of $\beta_{K}$ and the two correlations for $C_{f}$ and $\tilde{\pi}$ would not be as good if compared with the low-speed data (at low speeds $\beta=\beta_{K}$ ).

At least in the regions of adverse and zero pressure gradient it is concluded that the boundary-layer properties are insensitive to the upstream history (thus, the boundary layer is characterized by local conditions only) and that $\beta_{K}$ correlates low-speed data with both adiabatic and cold-wall high-speed data. As a final emphasis on this last conclusion, the velocity profiles at selected values of $\beta_{K}$ are compared for the three flows in figure 11, where the profiles are plotted in Clauser's defect form

$$
\begin{array}{ll}
\text { Clauser's defect form } & \frac{U_{e}^{*}-U^{*}}{U_{\tau}}=\mathrm{fn}\left(\frac{y}{\Delta} ; \beta_{K}\right), \\
\text { with } & \Delta \equiv \int_{0}^{\infty} \frac{U_{e}^{*}-U^{*}}{U_{\tau}} d y .
\end{array}
$$

\section{Experimental results for the recovery of a high-speed boundary layer after a step change in wall temperature}

In this study the inner pressure-generating centre body was removed from the model, which resulted in the development of a constant-pressure boundary along the model. As explained previously, liquid nitrogen was pumped through either 


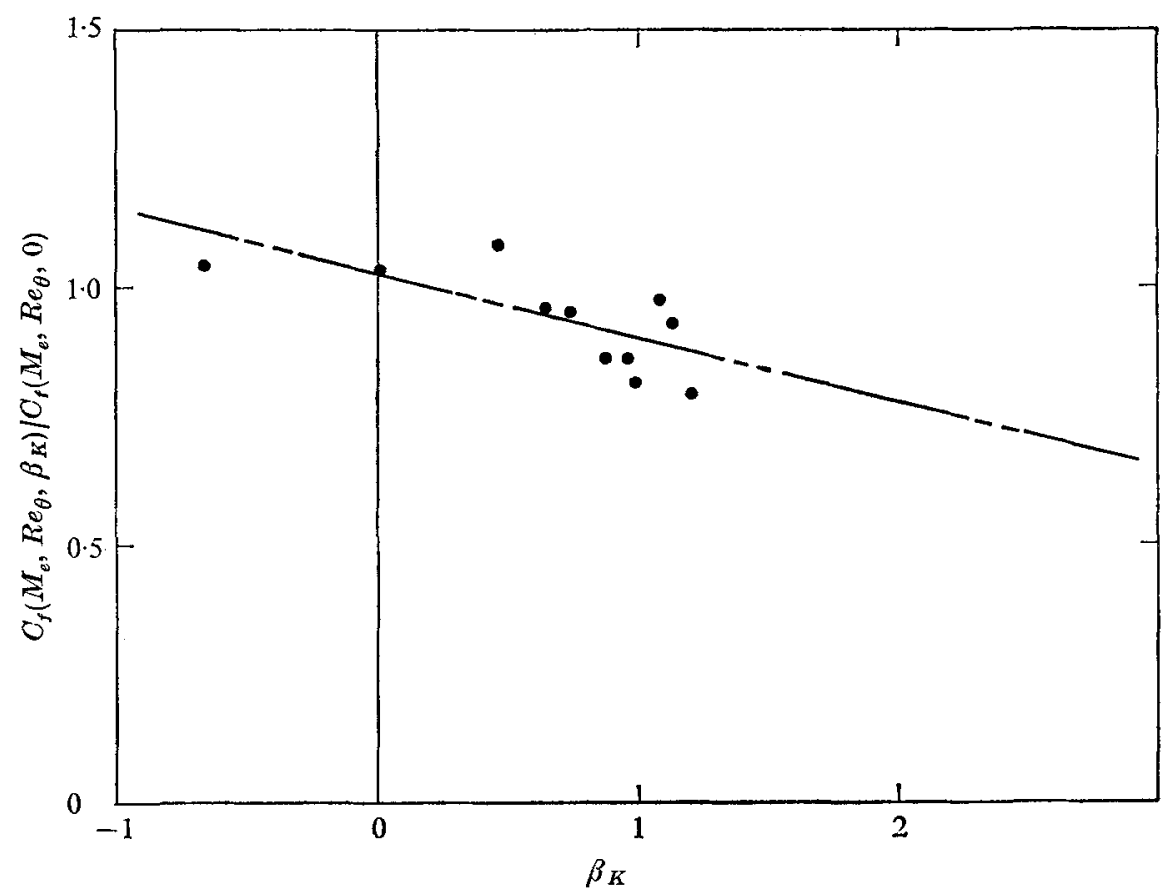

(a)

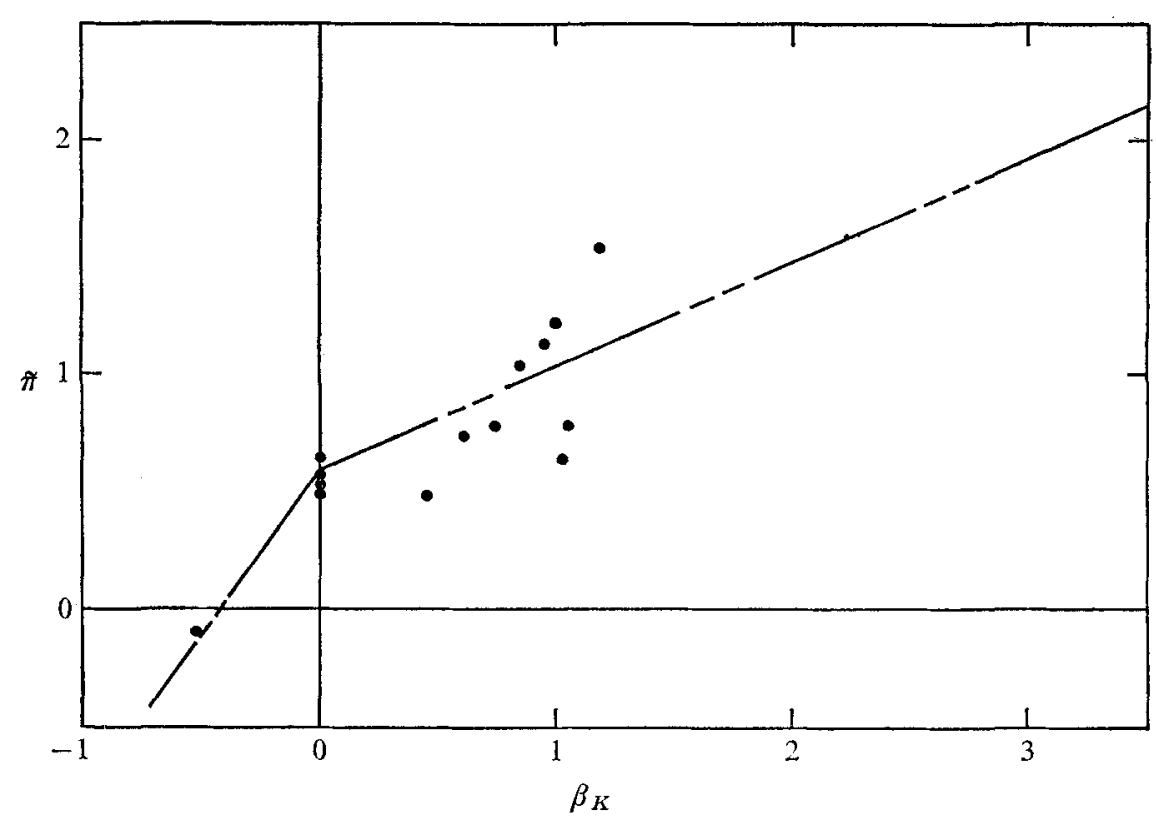

(b)

FIGURE 10. Effect of pressure gradient on $(a)$ skin-friction coefficient and $(b)$ wake strength

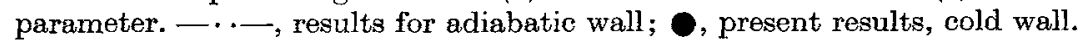



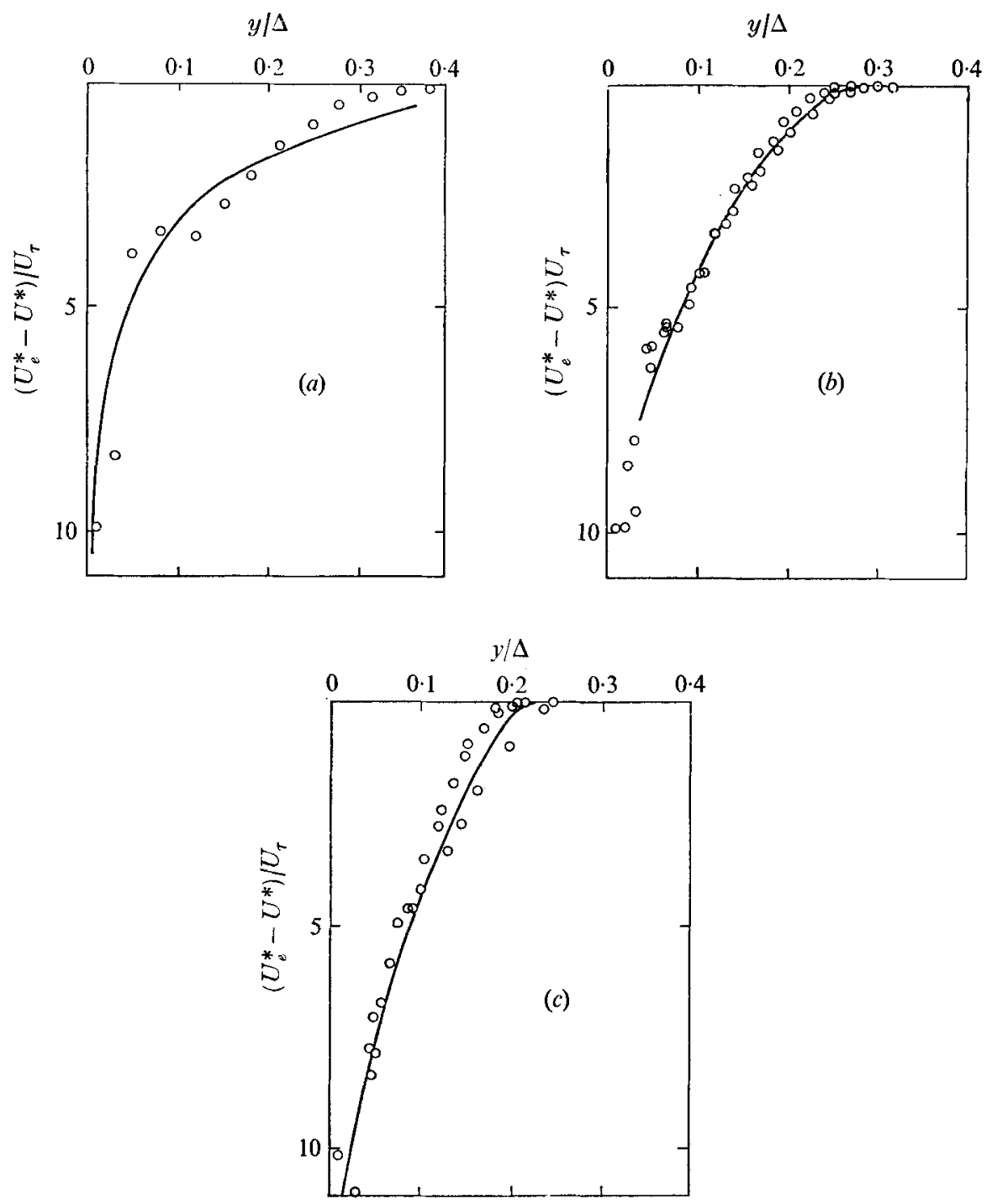

Figure 11. Transformed velocity profiles: Clauser defect form. (a) $\beta_{K}=-0 \cdot 5$, (b) $\beta_{K}=0,(c) \beta_{K} \approx 1$. - , adiabatic wall and low-speed data.

the fore or aft set of cooling coils while water at room temperature was pumped through the other set. This resulted in a sudden change in wall temperature $(\Delta x / \delta \approx 4)$ at $x=24$ in. as shown in figure $12 . \dagger$

Inasmuch as there is little difference between the velocity profiles of an adiabatic or cold-wall boundary layer, the primary measurement consisted of determining the rate of readjustment of the thermal structure within the existing turbulent boundary layer. Two cases were studied as indicated by figure 12: first,

$\uparrow$ This figure indicates the measured wall temperature distribution along the model. As explained, the wall temperature 'seen' by the boundary layer is inferred to be $0.50 T_{T_{e}}$ as opposed to the measured value of $0.30 T_{T_{e}}$. 

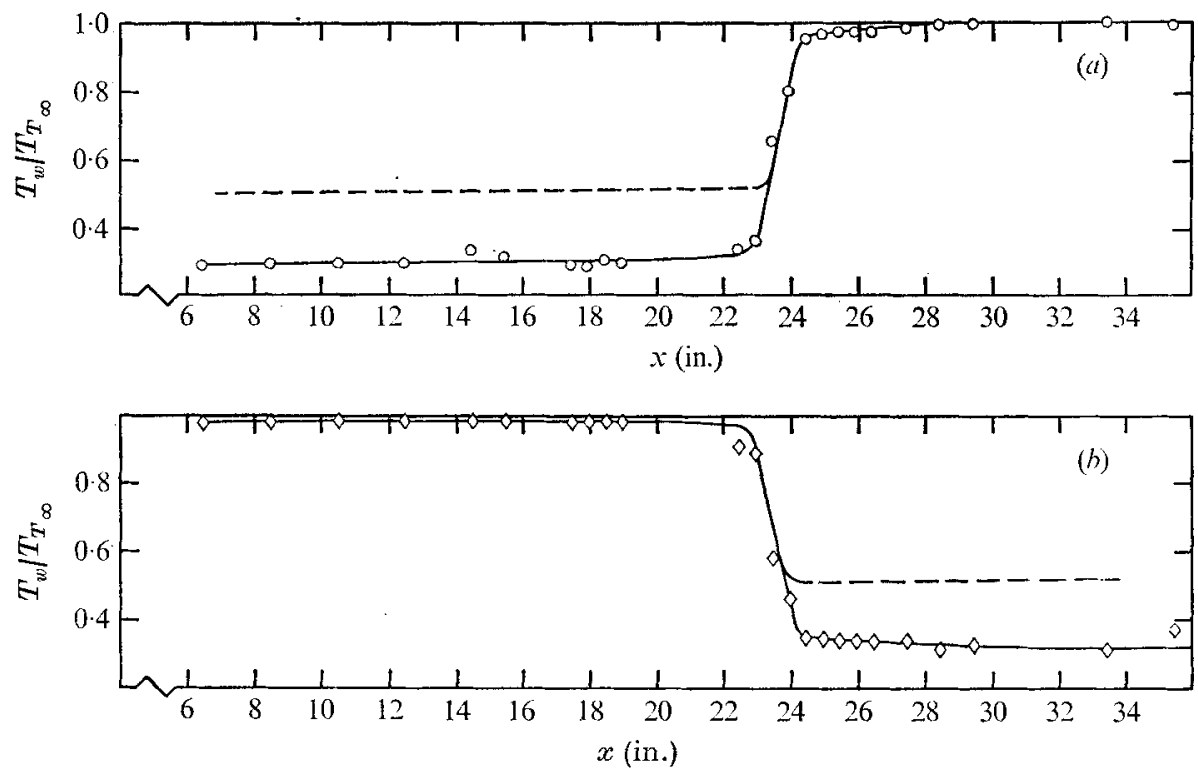

Figure 12. Measured wall temperature distributions. (a) Wall temperature 'step up'. (b) Wall temperature 'step down'. ___, fairing of data points; -....., estimated frost tomperature. $M=4 \cdot 0, R e=0.5 \times 10^{6} \mathrm{in}^{-1}, T_{R}=0.92$.

the 'step down' case where an initially adiabatic boundary layer flows onto a cold wall and second, the 'step up' case where an initially cooled boundary layer flows onto a wall near the recovery temperature.

One means of determining the thickness of the readjusting thermal boundarylayer thickness $\delta_{T}$ would be to plot profiles of $T_{T}[y / \delta(x) ; x]$ and to define $\delta_{T}$ as that value of $y$ where an observable deviation from $T_{T}[y / \delta(0) ; 0]$ is detected. This procedure, however, does not display the readjustment either graphically or accurately and hence, an alternative procedure was used. This procedure involves plotting the total-temperature data against the velocity data in the Crocco format

$$
\frac{T_{T}-T_{\mathrm{rep}}}{T_{T e}-T_{\text {rep }}}\left(\frac{U}{U_{e}} ; x\right)
$$

at each survey station, thus eliminating the subjective selection of $\delta(x)$. Further, in this form it is easier to visualize the readjustment from the initial totaltemperature distribution to the final distribution. $\dagger$

An example of such a plot is given in figure 13 for this step-up case. Here the reference temperature $T_{\text {ref }}$ is taken as the wall temperature for $x<0$ in order to keep the normalized total temperatures $O(1)$. Shown with each profile is a line representing the profile at $x=0$ and a tick mark where the total-temperature profile deviates from the initial profile by $0 \cdot 01\left(T_{T e}-T_{\text {ref }}\right)$ (this corresponds to a change of $2{ }^{\circ} \mathrm{C}$, which is more than twice the probe resolution).

$\dagger$ A third possible method of selecting $\delta_{T}$ based upon following flow streamlines

$$
\psi=\text { constant }=\int_{0}^{y(x)} \rho u d y
$$

was rejected because of the poor total-temperature resolution very close to the wall. 


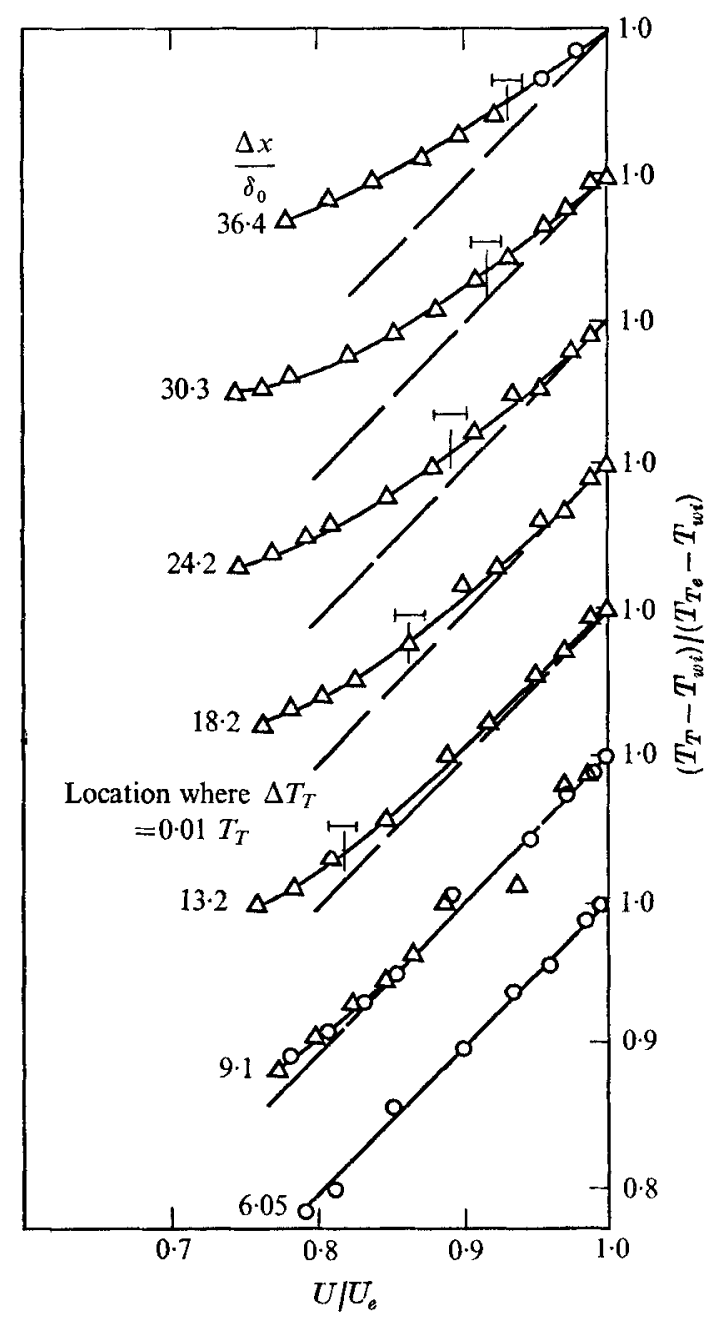

Figure 13. Total-temperature measurements: temperature step up. .....-, initial profile; _ _ fairing of data.

The value of $y$ corresponding to this choice of $U / U_{e}$ was taken as the thermal boundary-layer thickness $\delta_{T}$ at the station indicated. The distribution of $\delta_{T} / \delta_{0}$, where $\delta_{0}$ is the boundary-layer thickness at the temperature step location, is shown in figure $14(a)$ for the 'step-up' case. A similar plot for the 'step-down' case is shown in figure $14(b)$ evaluated in a manner similar to the above (the value of $\delta_{0}$ is the same for this configuration). Also shown in each of these figures is the boundary-layer thickness $\delta(x) / \delta_{0}$ and estimates of the viscous sublayer thickness $\left[y / \delta_{0}=50 /\left(\delta_{0} U_{\tau} / v_{w}\right)\right]$ ahead of and downstream of the change in wall temperature.

For the step-up case (an initially cold wall with the temperature changing to a value near the recovery temperature) the thermal boundary-layer thickness is observed to grow linearly with distance downstream of the temperature step. 


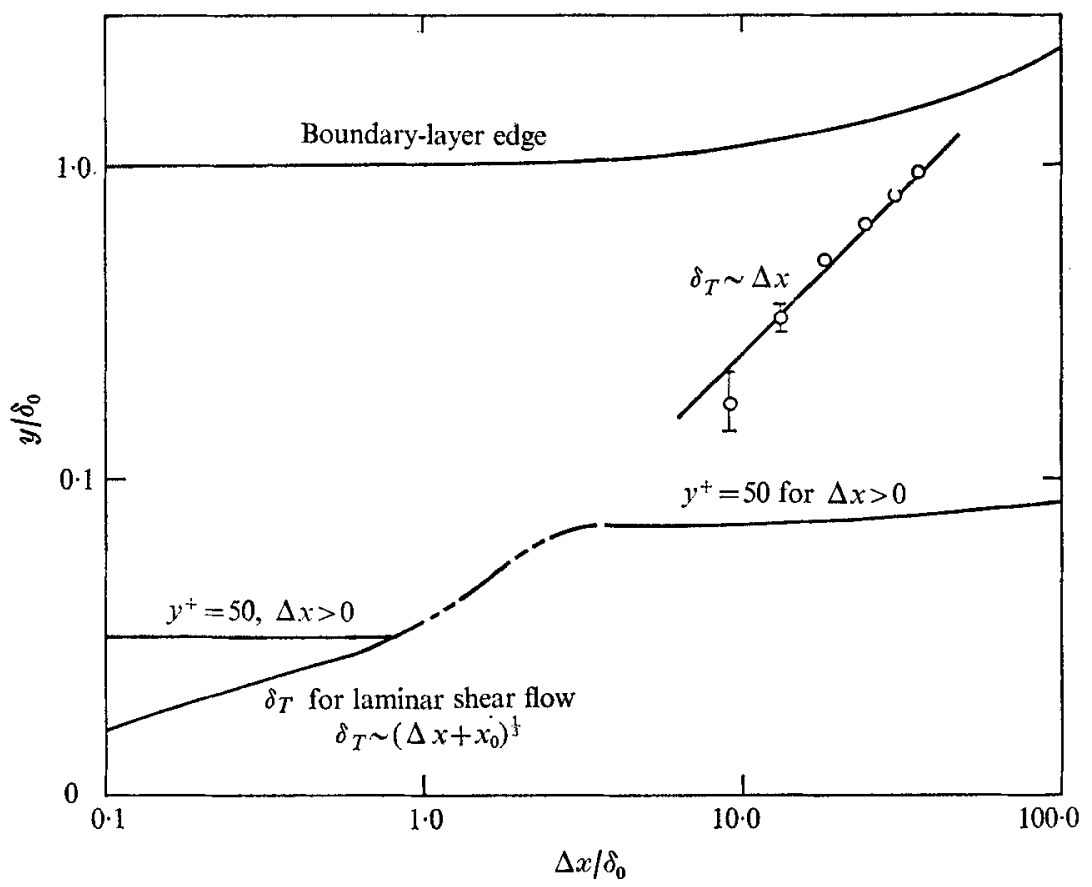

(a)

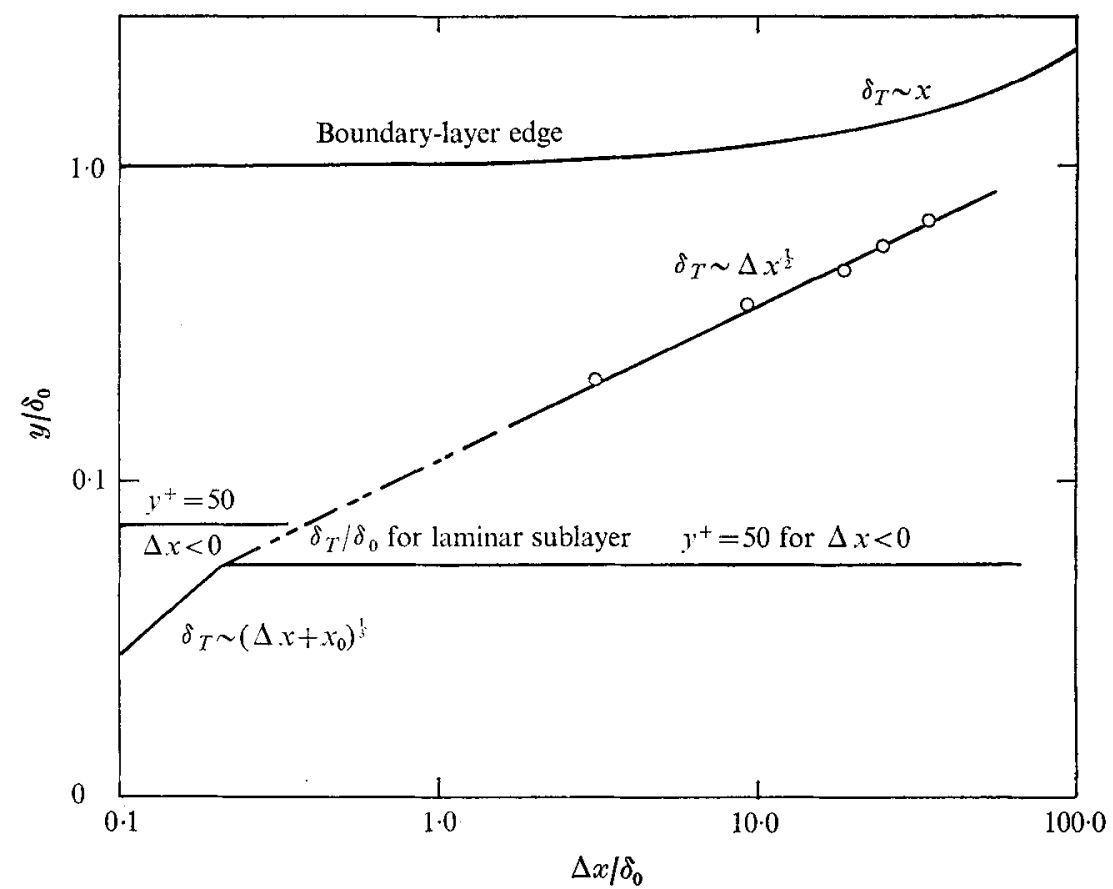

(b)

FIgURE 14. Thermal boundary-layer growth. (a) Step-up case. (b) Step-down case. 
This is in good agreement with the prediction by Townsend (1965) for low-speed boundary-layer flows. In contrast to this, however, the step-down case (initially adiabatic changing to a 'cold' wall) exhibits a growth rate of $\boldsymbol{\delta}_{T} \sim(\Delta x)^{\frac{1}{2}}$, which is interpreted to be a more laminar-like behaviour. Finally, it appears that in this latter case (step down in temperature) $\delta_{T}$ begins growing much sooner.

\subsection{Discussion}

Immediately downstream of the wall temperature change, the thermal boundary layer must first grow in thickness through the viscous sublayer before the fully turbulent growth takes place in the outer portion of the undisturbed boundary layer. As an estimate for the streamwise distance at which the thermal structure emerges from the viscous sublayer, the problem of a thermal boundary layer growing within an incompressible flow with a velocity field $u(y)=\left(\tau_{w} / \rho_{w}\right)^{\frac{1}{2}} y$ was addressed. For this idealized problem the solution for the thermal boundary-layer thickness is easily shown to be

$$
\delta_{T}(x) \approx 2 \cdot 6\left[\nu^{2} x / U_{\tau}^{2} \operatorname{Pr}\right]^{\frac{1}{3}},
$$

where $\operatorname{Pr}$ is the molecular Prandtl number $C_{p} \mu / k(\simeq 0 \cdot 7$ for air). It we estimate the value of $\delta_{T}(x)$ based upon the downstream wall conditions (i.e. skin friction and fluid properties) and determine where $\left(U_{\tau} / \nu_{w}\right)_{0} \delta_{t}=50$ for the step-up case, a value of $\Delta x / \delta_{0}=0.8$ is found, which is near the extrapolation of the $\delta_{t}(x)$ curve measured in the outer flow (see figure 14). For the step-down case a local value of $y^{+}=50$ is reached at $\Delta x / \delta_{0} \doteqdot 0 \cdot 1$, which is smaller than the distance to where the thermal boundary-layer structure thickness would grow to the initial sublayer thickness. Thus, turbulence can be generated within the original sublayer and the thermal structure will appear to originate sooner than in the step-up case.

Antonia \& Luxton have also observed different growth rates for a boundary layer flowing onto or off a rough wall. In their case the smooth-to-rough growth rate gave $\delta_{i} \sim x^{0.79}$, whereas the rough-to-smooth growth rate was observed to be $\delta_{i} \sim x^{0.43}$. The difference was attributed there to a higher turbulence intensity over the rough wall region. The distinct difference in growth rates observed by Antonia \& Luxton is very much like that observed here (although the present rates are somewhat higher).

There does not appear to be a one-to-one correspondence between these experiments. For example, one could interpret the temperature step-down case as equivalent to the low-speed smooth-to-rough case on the basis that the skinfriction coefficient increases with a decrease in wall temperature. However, a lower growth rate for the step-down case was observed whereas the corresponding smooth-to-rough case of Antonia \& Luxton gave a larger growth rate. Evidently, some other mechanism associated with the change in wall temperature (other than a change in $C_{f}$ ) is present. 


\section{Conclusions}

For supersonic $\left(M_{e} \lesssim 4\right)$ turbulent boundary layers with wall cooling $\left(T_{w} \approx 0.5 T_{T e}\right)$ in mild adverse pressure gradients, the velocity profiles are in good agreement with low-speed results when transformed according to Van Driest. In a region of adverse pressure gradient $\left(\beta_{K} \lesssim 1\right)$ the boundary layer was found to be in local equilibrium (i.e. characterized by the local value of $\beta_{K}$ instead of exhibiting upstream history effects). The effects of the pressure gradient (characterized by $\beta_{K}$ ) on the skin friction and wake strength parameter $\tilde{\pi}$ were found to agree with adiabatic-wall high-speed data and with low-speed data.

For a constant-pressure supersonic boundary layer subjected to a near step change in wall temperature the readjustment of the thermal structure was found to grow like $x$ for the case of an initially cooled wall boundary layer flowing onto a wall near the recovery temperature. For the opposite case where an adiabatic boundary layer flows onto a cold wall $\left(T_{w} \approx 0.5 T_{R}\right)$ the thermal-structure thickness was found to grow like $x^{\frac{1}{2}}$. Further, the apparent shift in the origin of growth for these two cases was attributed to the difference in the sublayer thicknesses and the growth rate within the sublayer.

The authors gratefully acknowledge the support of the Advanced Research Projects Agency and the U.S. Air Force Space and Missile System Organization under Contract F04701-71-C-0040 and the co-operation of the staff of the von Kármán Gas Dynamics Facility at AEDC, where the experiments were performed.

\section{REFERENCES}

Alber, I. E. \& Coats, D. E. 1969 Analytical investigation of equibrium and nonequilibrium compressible turbulent boundary layers. A.I.A.A. Paper no. 69-689.

Antonia, R. A. \& Luxton, R. E. 1971 The response of a turbulent boundary layer to a step change in surface roughness. Part 1. Smooth to rough. J. Fluid Mech. 48, 721-761.

Antonia, R. A. \& Luxton, R. E. 1972 The response of a turbulent boundary layer to a step change in surface roughness. Part 2. Rough to smooth. J. Fluid Mech. 53, 737-757.

Bird, R. B., Stewart, W. E. \& Lightfoot, E. N. 1963 Transport Phenomena. Wiley.

ChEN, K. K. 1972 Compressible turbulent boundary layer heat transfer to rough surfaces in pressure gradient. A.I.A.A. J. 10, 623-635.

Clauser, F. H. 1956 The turbulent boundary layer. Adv. in Appl. Mech. 4, 1-51.

Coles, D. E. 1953 Measurements in the boundary layer on a smooth flat plate in supersonic flow. 1. The problem of the turbulent boundary layer. Jet Propulsion Lab. Rep. no. 20-69.

Cotes, D. E. 1956 The law of the wake in the turbulent boundary layer. J. Fluid Mech. 1, 191-226.

Coles, D. E. \& Hirst, E. A. 1969 Proc. Computation of Turbulent Boundary Layers, 1968 AFOSR-IFP-Stanford Conf. vol. 2. Thermosciences Division, Stanford University, California.

HAEN, J. S. \& LUtz, R. G. 1971 Experimental investigation of turbulent boundary layers with pressure gradient and heat transfer at Mach number 4. Arnold Engng Development Centre Rep. AEDC-TR-71-3. 
Hopkins, E. J. \& INouYe, M. 1971 An evaluation of theories for predicting turbulent skin friction and heat transfer on flat plates at supersonic and hypersonic Mach numbers. A.I.A.A. J. 9, 993-1003.

LEwrs, J. E., Gran, R. L. \& Kubota, T. 1972 An experiment on the adiabatic compressible turbulent boundary layer in adverse and favourable pressure gradients. $J$. Fluid Mech. 51, 657-672.

Matse, G. \& McDonatd, H. 1967 Mixing length and kinematic eddy viscosity in a compressible boundary layer. A.I.A.A. 5th Aerospace Sci. Meeting.

Matthews, D. C., Ghildos, M. E. \& Paynter, G. C. 1970 Use of Coles' universal wake function for compressible turbulent boundary layers. J. Aircraft, 7, 137-140.

Mellor, G. L. \& Gibson, D. M. 1966 Equilibrium turbulent boundary layers. J. Fluid Mech. 24, 225-253.

Townsend, A. A. 1965 Self-preserving flow inside a turbulent boundary layer. $J$. Fluid Mech. 22, 773-797.

VAN DRIEST, E. R. 1951 The turbulent boundary layer in compressible fluids. J. Aero Sci. $18,145-160$.

Wegner, P. P. \& Mack, L. M. 1958 Adv. in Appl. Mech. 5, 307-447. 